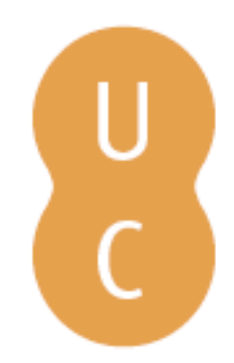

\title{
pompalina
}

\section{Um modelo para a Bibliotheca do Povo e das Escolas: a Biblioteca del Popolo}

\author{
Author(s: Bonifácio, Vitor \\ Published by: $\begin{aligned} & \text { Imprensa da Universidade de Coimbra; UA Editora - Universidade de } \\ & \text { Aveiro }\end{aligned}$
}

Persistent URL: URI:http://hdl.handle.net/10316.2/46171

DOI: $\quad$ DOl:https://doi.org/10.14195/978-989-26-1711-4_9

Accessed : $\quad$ 16-Sep-2020 17:13:41

The browsing of UC Digitalis, UC Pombalina and UC Impactum and the consultation and download of titles contained in them presumes full and unreserved acceptance of the Terms and Conditions of Use, available at https://digitalis.uc.pt/en/terms_and_conditions.

As laid out in the Terms and Conditions of Use, the download of restricted-access titles requires a valid licence, and the document(s) should be accessed from the IP address of the licence-holding institution.

Downloads are for personal use only. The use of downloaded titles for any another purpose, such as commercial, requires authorization from the author or publisher of the work.

As all the works of UC Digitalis are protected by Copyright and Related Rights, and other applicable legislation, any copying, total or partial, of this document, where this is legally permitted, must contain or be accompanied by a notice to this effect. 


\title{
UM MODELO PARA A BIBLIOTHECA DO POVO E DAS ESCOLAS: A BIBLIOTECA DEL POPOLO
}

\author{
A MODEL FOR THE BIBLIOTHECA DO POVO \\ E DAS ESCOLAS: THE BIBLIOTECA DEL POPOLO
}

Vitor Bonifácio ${ }^{1}$

Resumo: Entre 1881 e 1913 publicaram-se 237 números da Bibliotheca do Povo e das Escolas (BPE). Fruto de uma eficaz estratégia comercial, esta aposta do editor David Corazzi (1845-1896) obteve um enorme sucesso no espaço geográfico de língua portuguesa. Os dezasseis números iniciais tiveram primeiras edições com tiragens iguais ou superiores a 10000 exemplares, um facto extraordinário no panorama editorial português da época. Concetualmente, a publicação da BPE enquadra-se nas dinâmicas editoriais europeias da segunda metade do século XIX. O desenvolvimento de novas tecnologias de impressão permitiu uma descida do preço das publicações, nomeadamente das periódicas: jornais, revistas e fascículos. A perceção generalizada da necessidade

${ }^{1}$ Centro de Investigação "Didática e Tecnologia na Formação de Professores", Departamento de Física da Universidade de Aveiro: vitor.bonifacio@ua.pt. Este trabalho é financiado por Fundos Nacionais através da FCT - Fundação para a Ciência e a Tecnologia, I.P., no âmbito do projeto UID/CED/00194/2013. 
de providenciar instrução às classes populares implicou um incremento da alfabetização e dos sistemas públicos de ensino, em particular, do primário, e, consequentemente, do número de possíveis leitores. Quer o editor, quer Xavier da Cunha (1840-1920), o primeiro diretor da coleção, referiram que se inspiraram em modelos estrangeiros sem, no entanto, os especificarem. Até agora estas afirmações têm sido interpretadas como significando a utilização de uma estratégia editorial globalmente semelhante à de outras publicações, isto é, volumes educativos de baixo custo organizados numa "biblioteca". Neste artigo argumentamos que a Biblioteca del Popolo, publicada a partir de 1875, em Milão, pelo editor Edoardo Sonzogno (1836-1920) constituiu, de facto, o modelo da BPE. Concluímos que as especificidades da publicação portuguesa refletem uma opção editorial que definiu como alvos privilegiados os mercados brasileiro e do livro escolar. Palavras-chave: História do Livro; Bibliotheca do Povo e das Escolas; Biblioteca del Popolo; Edoardo Sonzogno

Abstract: The two hundred and thirty seven volumes of the Bibliotheca do Povo e das Escolas (People and Schools Library, BPE) were published between 1881 and 1913. The publisher David Corazzi (1845-1896) savvy commercial strategy helped the publication to attain an immense success in Portugal and Brazil. The first sixteen numbers had print runs of 10000 copies or more. At the time these numbers were unusually high in Portugal. Conceptually the BPE falls in line with international publishing dynamics of the second half of the nineteenth century. The development of new printing technologies led to a price decrease of printed materials, namely that of periodicals: newspapers, magazines and fascicles. The widespread belief that popular classes needed instruction 
implied an increased investment in public education, particularly at the primary level. Naturally higher literacy led to a larger number of potential readers. Both the publisher and Xavier da Cunha (1840-1920), the first editor of the collection, claimed that foreign models inspired the creation of BPE. Notwithstanding neither referred a specific model. So far these statements have been interpreted to mean that an editorial strategy similar to other publications was implemented, i.e. low cost educational volumes organized into a virtual library. In this article we argue that the Biblioteca del Popolo, published from 1875 onwards, in Milan, by the publisher Edoardo Sonzogno (1836-1920) constituted, in fact, the BPE model. We further conclude that the specificities of the Portuguese publication reflect an editorial strategy targeting the Brazilian and educational markets.

Keywords: Book History; Bibliotheca do Povo e das Escolas; Biblioteca del Popolo; Edoardo Sonzogno 


\section{Introdução}

Durante o século XIX assiste-se, em geral, a uma expansão do mercado do livro e, em particular, do livro de divulgação. O aparecimento das máquinas de impressão rotativas, de novas técnicas de fabrico de papel e impressão possibilitaram o aumento das tiragens e a oferta de publicações a preços inferiores ${ }^{2}$. Simultaneamente, diferentes contextos ideológicos privilegiaram a instrução enquanto principal agente de transformação dos cidadãos. Segundo Torgal, durante o liberalismo português: "Afirmar-se-á insistentemente que é a ignorância que torna os homens perversos e que a educação e instrução são a base do edifício social"3. A viabilidade das novas ideias políticas encontrava-se dependente dos cidadãos. Nas palavras de Manuel Pinheiro Chagas (1842-1895),

da mesma forma que os soberanos absolutos cometiam um verdadeiro crime não educando convenientemente os seus herdeiros, a quem ainda um dia haviam de ser confiados os destinos de milhões de homens, assim os governos liberais cometem o mesmo crime, quando não promovem a instrução do povo que é hoje o verdadeiro soberano, que chegou neste século à maioridade, que tem enfim nas suas mãos a direção dos seus próprios destinos ${ }^{4}$.

Ao mesmo tempo, o desenvolvimento tecnológico criou novas profissões, por exemplo, nas áreas das comunicações e

${ }^{2}$ Ernst HOMBURG, "From Chemistry for the People to the Wonders of Technology: The Popularization of Chemistry in the Netherlands during the Nineteenth Century": Hyle - International Journal for Philosophy of Chemistry 12(2) (2006), pp. 163-191.

${ }^{3}$ Luís Reis TORGAL, "A instrução pública", in José MATTOSO (ed.), História de Portugal, Vol. 5. Lisboa, Círculo de Leitores, 1993, p. 609.

${ }^{4}$ Citado por António Pedro Barbas HOMEM, Manuel Joaquim Pinheiro Chagas, 1842-1895: uma biografia. Lisboa, Colecção Parlamento, Assembleia da República, 2012, p. 27. 
da iluminação. A necessidade de qualificar estes profissionais implicou a criação de sistemas de instrução especializados, como o Instituto Industrial de Lisboa, e a disponibilidade de manuais e livros de instrução. O desenvolvimento económico e a instrução eram, para as elites da época, inseparáveis5. Todos estes fatores contribuem, assim, para a expansão, ao longo do século XIX, dos sistemas públicos de ensino, nomeadamente do primário ${ }^{6}$.

Os novos leitores constituíam um mercado atraente para os editores que passam a oferecer um número crescente de materiais impressos, iniciando o que se convencionou designar por "cultura de massas" alheios à condição social de leitores com baixo poder económico, reduzido número de horas de lazer e sem hábitos de frequência de livrarias ${ }^{8}$. Uma estratégia habitual consistia em comercializar as obras por fascículos. A subdivisão do livro distribuía o esforço de aquisição por um maior intervalo de tempo, permitia que cada fascículo tivesse um preço acessível e proporcionava uma solução prática para a escassez de tempo de leitura9 9 .

5 Ana Cardoso de MATOS, "Os agentes e meios de divulgação científica e tecnológica em Portugal no século XIX": Scripta Nova 69 (2000). Disponível em linha: http://www.ub.edu/geocrit/sn-69-29.htm (consultado a 15/06/2018).

${ }^{6}$ Martyn LYONS, "New Readers in the Nineteenth Century: Women, Children, Workers”, in Guglielmo CAVALLO, Roger CHARTIER (eds.), A History of Reading in the West. Cambridge, Blackwell, 2003, pp. 313-344.

7 S. SHEETS-PYENSON, "Popular Science Periodicals in Paris and London: the Emergence of a Low Scientific Culture, 1820-1875": Annals of Science 42(6) (1985), pp. 549-572; Bruno BÉGUET, "La vulgarisation scientifique au XIX ${ }^{\mathrm{e}}$ siècle", in Bruno BÉGUET, Maryline CANTOR, Ségolène LE MEN (eds.), La Science pour Tous. Paris, Réunion des Musées Nationaux, 1994, pp. 5-48; Bernard LIGHTMAN, "The Voices of Nature: Popularizing Victorian Science", in Bernard LIGHTMAN (ed.), Victorian Science in Context. Chicago, The University of Chicago Press, 1997, pp. 187-211; M. LYONS, "New Readers...", op. cit.

8 M. SANTOS, "A elite intelectual e a difusão do livro nos meados do século XIX”: Análise Social XXVII (116-117) (1992), pp. 539-546.

9 Jean-Yves MOLLIER, "La construction du système éditorial français et son expansion dans le monde du XVIII ${ }^{\mathrm{e}}$ au $\mathrm{XX}^{\mathrm{e}}$ siècle", in Jacques MICHON, Jean-Yves 
A publicação da edição francesa de O Capital exemplifica as vantagens atribuídas à venda por fascículos. Segundo o editor, Maurice Lachatre,

o método de publicação adotado, por fascículos de dez cêntimos, terá a vantagem de permitir a um número maior dos nossos amigos de obter o seu livro, os pobres não podem pagar pela ciência senão pouco dinheiro; o seu objetivo será concretizado: tornar o seu trabalho acessível a todos ${ }^{10}$.

Karl Marx (1818-1883), numa carta enviada de Londres, em março de 1872, aplaude a ideia: "Desta forma a obra será mais acessível à classe trabalhadora, uma consideração que, para mim, supera tudo o resto ${ }^{11}$." A comercialização em fascículos permitia, ainda, utilizar a rede de distribuição de jornais, aproximando o impresso das classes populares ${ }^{12}$. O sucesso obtido pela Librarie Hachette com a disponibilização das suas coleções nas "bibliothèques" das gares dos caminhos de ferro ilustra as vantagens da presença do livro na "rua"13. Por último, a venda em fascículos cria colecionadores, isto é, estimula hábitos de compra resultantes do desejo de completar as obras. Não será, por isso, de estranhar que simultaneamente apareçam à venda coleções designadas por "bibliotecas". A atenção dada ao título da série

MOLLIER (eds.), Les mutations du livre et de l'édition dans le monde du XVIII siècle à l'an 2000. Paris, L'Harmattan, 2003, pp. 47-72.

10 Karl MARX, Le capital. Paris, M. Lachâtre, 1872. As traduções efetuadas são da responsabilidade do autor deste artigo.

${ }^{11}$ Karl MARX, Le capital, op. cit.

12 Anne-Marie THIESSE, Le roman du quotidian. Paris, Éditions du Seuil, 2000, p. 21.

13 Frédéric BARBIER, "Libraires et colporteurs", in Roger CHARTIER, Henri-Jean MARTIN (eds.), Histoire de l'édition française, Vol. 3. Paris, Fayard, Cercle de la Librarie, 1990, pp. 256-302. 
ajudava a que cada texto constituinte aparecesse como parte integrante de um programa extenso e cuidadosamente concebido; e, do ponto de vista da rentabilidade, criava a expectativa de que o aumento sistemático do conhecimento do tema (mesmo que amplo e por vezes inteiramente artificial) pudesse ser, de facto, adquirido pela leitura de todos os volumes ${ }^{14}$.

No caso da BPE cada número pode ser interpretado como um fascículo de um livro - a série. O conjunto das séries constituiria a biblioteca.

A investigação indica que a edição portuguesa, durante o século XIX, não se diferenciou, no essencial, das dinâmicas internacionais. Note-se, contudo, que a baixa literacia, "uma ilha de letrados num mar de analfabetos" de uma população pequena, não tenha possibilitado, em muitos casos, a existência de uma massa crítica capaz de sustentar no tempo muitos dos esforços editoriais efetuados ${ }^{15}$. Na divulgação da astronomia, por exemplo, encontram-se iniciativas pontuais de curta duração e/ou não concluídas ${ }^{16}$. Neste sentido, a BPE apresenta-se como uma exceção, quer pela longevidade da publicação, quer pelas elevadíssimas tiragens de alguns dos seus números ${ }^{17}$.

\footnotetext{
${ }^{14}$ Iain BEAVAN, "Advertising Judiciously: Scottish Nineteenth-Century Publishers and the British Market", in Peter ISAAC, Barry MCKAY (eds.), The Mighty Engine: The printing Press \& its Impact. Winchester, St Paul's Bibliographies and Delaware, Oak Knoll Press, 2000, p. 73.

15 José Murilo de Carvalho citado por A. P. B. HOMEM, op. cit., p. 26.

16 Vitor BONIFÁCIO, Da Astronomia à Astrofísica: A perspectiva portuguesa. Tese de Doutoramento, Universidade de Aveiro, Departamento de Física, 2009.

17 Manuela D. DOMINGOS, Estudos de Sociologia da Cultura. Livros e Leitores do Século XIX. Lisboa, Instituto Português do Ensino à Distância, 1985, p. 13.
} 


\section{A Bibliotheca do Povo e das Escolas}

David Corazzi (figura 1) publica, em fevereiro de 1881, em Lisboa, o primeiro número da BPE, Historia de Portugal. Segundo o editor, a coleção enquadrava-se na "tendencia para a vulgarização dos conhecimentos humanos" presente nas sociedades modernas e destinava-se a providenciar instrução aos "que não sabem por thes fallecerem recursos com que apprendam", ou seja, às classes populares ${ }^{18}$ :

A Bibliotheca do Povo e das Escolas vem acudir a uma falta que já, desde tempos, outros paizes taes como a Inglaterra, a França, a Itália, a Allemanha e os Estados-Unidos da America, têem tratado de remediar dando a publico, por modico preço, collecções no genero da que ora sai a lume ${ }^{19}$.

Xavier da Cunha (figura 1), diretor da coleção, entre 1881 e 1894, refere igualmente a "inspiração" de modelos estrangeiros. Nas "Duas palavras ao leitor”, anexas à encadernação da 3. série, escreve que tomou "por modelos de fórma as collecções analogas publicadas em França, na Italia e em outros paizes que marcham na vanguarda da civilização" 20 .

18 Xavier da CUNHA, "Quatro paginas de prólogo", in Biblioteca do Povo e das Escolas. Sexta Serie. Lisboa, David Corazzi - Editor. Empreza Horas Romanticas, 1883.

19 X. da CUNHA, "Quatro páginas...", op. cit.

20 Xavier da CUNHA, "Duas palavras ao leitor", in Biblioteca do Povo e das Escolas. Terceira Serie. Lisboa, David Corazzi - Editor. Empreza Horas Romanticas, 1882 . 

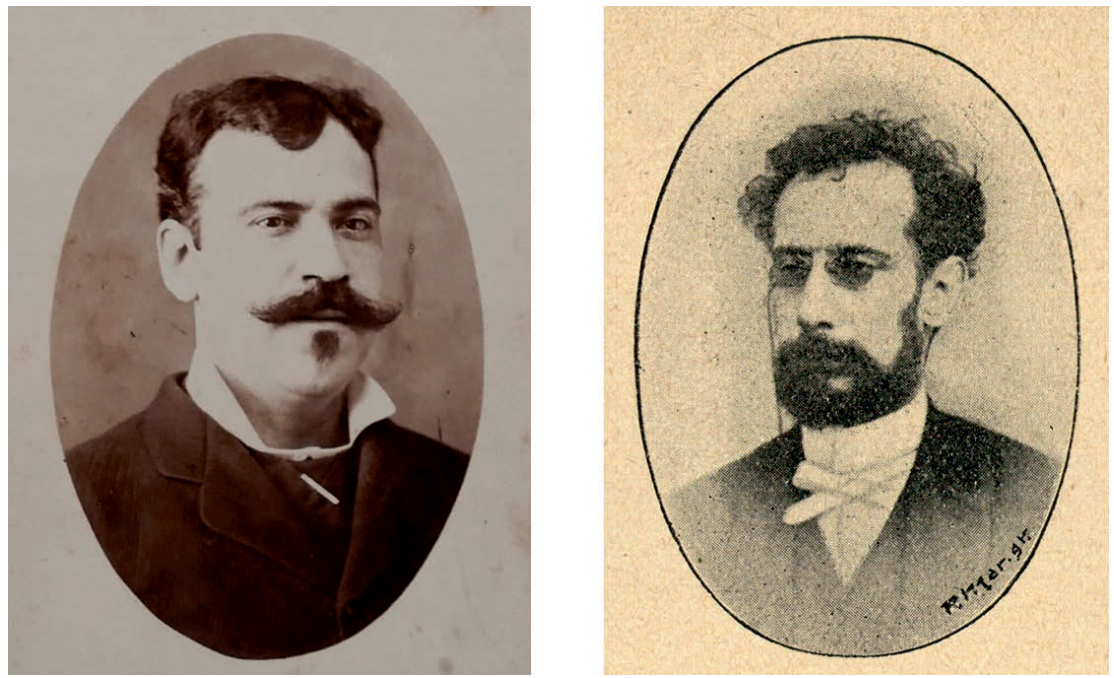

Figura 1 - Retratos de David Corazzi ${ }^{21}$ e Xavier da Cunha 22 , imagens da esquerda e direita, respetivamente

Infelizmente, nem Corazzi nem Cunha especificam o(s) modelo(s) utilizado(s). Manuela Domingos, no exaustivo estudo que dedicou à BPE, não apresentou um modelo específico da coleção, argumentando, contudo, contra a possibilidade de esta se inspirar nos Manuels Roret. Publicados, em França, a partir da década de 20, os manuais Roret eram conhecidos e distribuídos em Portugal, mas "os seus preços eram evidentemente impopulares - 250 a 1000 réis cada volume - tal como a sua extensão - 300 a 400 páginas"23. Valores que contrastam com o custo de 50 réis de cada número de 64 páginas, incluindo capas, da BPE. Trabalhos posteriores

21 Magalhães LIMA, "David Corazzi": Commercio e Industria - Sciencias, Artes e Letras 6 (1880), p. inum.

22 Imagem cedida pela Hemeroteca Municipal de Lisboa. ANÓNIMO, "Comemorações do XXV anno do Occidente": O occidente: revista illustrada de Portugal e do estrangeiro XXV (829-830, 10 e 20 de Janeiro) (1902), p. 7.

23 M. DOMINGOS, op. cit., p. 46; M. LYONS, “New Readers...”, op. cit., p. 443. 
prosseguiram outras linhas de investigação, ficando em aberto a questão de um hipotético modelo ${ }^{24}$.

Note-se que, apesar da inspiração estrangeira, Xavier da Cunha, o primeiro e talvez o único editor formal da BPE, considerava-se o "arquitecto" da coleção tendo-a estabelecido com

um plano mais vasto, mais desenvolvido, mais desdobrado, menos deficiente e (quando possivel) mais apurado e consciencioso do que o methodo seguido pelas collecções extrangeiras (algumas das quaes - triste é dizêl-o - chegam a admittir nas suas paginas verdadeira obra de fancaria ${ }^{25}$.

A publicação da BPE estende-se até 1913. Inicialmente, a coleção mantém a periodicidade bimensal prevista. Segundo o editor, os números seriam disponibilizados para venda nos dias 10 e 25 de cada mês. Entre 1886 e 1891 publicou-se, em média, um número por mês, enquanto os últimos 41 números são dados à estampa, de

${ }^{24}$ Jorge NASCIMENTO, "Nota prévia sobre a palavra impressa no Brasil no século XIX: a biblioteca do povo e das escolas": Horizontes 19 (2001), pp. 11-27; Maria de Fátima NUNES, "A Bibliotheca do Povo e das Escolas: Fonte para a História da Ciência", in Mari Álvarez LIRES, Ánxela Bugallo RODRÍGUEZ, José $M^{a}$ FERNANDÉZ, Rafael Sisto EDREIRA e X. Carlos Valle PÉREZ (coord.), Estudios de Historia das Ciencias e das Técnicas, vol. 1. Vigo, Deputacíon Provincial. Servicio de Publicacións, 2001, pp. 221-231; Giselle M. VENÂNCIO, "Lisboa-Rio de Janeiro-Fortaleza: os caminhos da coleção Biblioteca do Povo e das Escolas, traçados por David Corazzi, Francisco Alves e Gualter Rodrigues": Cultura 21 (2005), pp. 185-204; Jorge NASCIMENTO, V. SANTOS, "Geografia geral para portugueses e brasileiros: a biblioteca do povo e das escolas": Revista da Faced 10 (2006), pp. 141-158; Vitor BONIFÁCIO, Isabel MALAQUIAS, João FERNANDES, “Ernesto Vasconcellos' Astronomia Photographica: the earliest popular book on astronomical photography?": Journal of Astronomical History and Heritage 11 (2008), pp. 116-123; Vitor BONIFÁCIO, Da Astronomia à Astrofísica: A perspectiva portuguesa. Tese de Doutoramento, Universidade de Aveiro, Departamento de Física, 2009; Olímpia de J. de B. M. NABO, Educação e difusão da ciência em Portugal. A 'Bibliotheca do Povo e das Escolas' no Contexto das Edições Populares do Século XIX. Tese de Mestrado, Escola Superior de Educação do Instituto Politécnico de Portalegre, 2012; Vitor BONIFÁCIO, Helmuth MALONEK, "Os inesperados livros de Matemática da "Bibliotheca do Povo e das Escolas"”: Suplemento do Boletim da Sociedade Portuguesa de Matemática 69 (2013), pp. 35-37.

25 X. da CUNHA, "Duas palavras...", op. cit. 
forma irregular, ao longo de 22 anos $^{26}$. Cada número de $11 \mathrm{~cm}$ por $16,5 \mathrm{~cm}$ tinha 64 páginas e custava 50 réis, valor que, na altura, permitia adquirir cinco exemplares do jornal, de quatro páginas, Diario Illustrado, de Lisboa. O baixo preço dos números era amiúde referido, por vezes com ironia, nas recensões encontradas nos jornais:

"Astronomia Popular" - Assim se intitula o $10^{\circ}$ volume da Bibliotheca do Povo e das Escolas, publicação a todos os respeitos excellente e de que é editor o snr. David Corazzi. O dito volume, que recebemos, vem illustrado de 15 gravuras. Não esqueça que o preço de cada um ascende... a 50 réis $^{27}$ !

No início, os números eram publicados anonimamente. Xavier da Cunha identifica nos prólogos, a partir da $3 .^{a}$ série, os autores de cada número, à exceção dos escritos por si. Os três títulos escritos por José Júlio Rodrigues (1843-1923), publicados nas 11. e 13. a séries, são os primeiros a incluir o nome do autor na capa. Esta prática passa a ser norma a partir do número 105, da $14 .^{\mathrm{a}}$ série, de 1885.

A coleção foi inicialmente bem recebida, quer pela crítica, quer pelo público. Os dez mil exemplares da primeira edição da Historia de Portugal esgotaram em poucos dias, tendo a segunda edição de seis mil exemplares sido de imediato dada à estampa ${ }^{28}$. Atualmente conhecem-se apenas as tiragens dos dezasseis primeiros números. O n. ${ }^{\circ}$ 16, Hygiene, escrito pelo médico naval João Cesário Lacerda (1841-1903), teve uma primeira edição de vinte mil exemplares, um valor enorme no panorama editorial português da época ${ }^{29}$.

\footnotetext{
${ }^{26}$ M. DOMINGOS, op. cit., Quadro I, p. 29.

${ }^{27}$ ANÓNIMO, "Astronomia popular": O Primeiro de Janeiro, 13 de julho (1881), p. 2.

28 Xavier da CUNHA, Historia de Portugal, 2. ${ }^{a}$ edição. Lisboa, David Corazzi Editor - Empreza Horas Romanticas, 1881.

29 M. DOMINGOS, op. cit., p. 76; João Cesario de LACERDA, Hygiene. Lisboa, David Corazzi Editor - Empreza Horas Romanticas, 1881.
} 


\section{A Biblioteca del Popolo}

Edoardo Sonzogno (1836-1920) (figura 2) iniciou a sua atividade editorial, em 1861, através da publicação de uma revista ilustrada, Lo Spirito Folletto. Esta opção resulta, segundo Barile, não só da elevada taxa de analfabetismo, 70\% dos habitantes das cidades, mas também do baixo número daqueles que sabiam italiano, apenas 0,8\% da população ${ }^{30}$. Em 1865, Sonzogno dá à estampa outra revista ilustrada, L'Emporio pittoresco, destinada ao grande público. O baixo custo, o número e a qualidade das ilustrações levam a que, dois anos depois, a revista atinja tiragens de 60000 exemplares, um valor 20 vezes superior ao de outras revistas italianas contemporâneas ${ }^{31}$.

Sonzogno, após uma visita à Exposição Universal de Paris de 1867 , onde toma contacto com a prensa rotativa Marioniti, moderniza a impressão do jornal Il Secolo, fundado no ano anterior, e transforma-o no primeiro grande diário italiano. Tal como os seus congéneres franceses, Sonzogno utilizou como estratégia comercial a apetência popular pela leitura de romances. Em 1872, quando o jornal publicava dois e, por vezes, três folhetins por dia, Sonzogno torna-se o representante italiano da Société des gens des lettres, assegurando, assim, o direito de tradução dos romances franceses mais populares ${ }^{32}$. Consequentemente, Il Secolo atingiu, no final do século XIX, uma tiragem diária de 150000 exemplares $^{33}$. Por outro lado, os folhetins "alimentavam" outras iniciativas da editora, nomeadamente várias coleções de romances tais como a Biblioteca

30 Laura BARILE, Le parole illustrate: Edoardo Sonzogno editore del popolo. Ghirlandina. Incontri con l'Ottocento. Modena, Mucchi Editore, 1994, p. 10.

31 Laura BARILE, Le parole illustrate..., op. cit., p. 10.

32 Viola CAGNINELLI, "Edoardo Sonzogno rappresentante italiano della Société des gens de lettres (1872-1878)": La fabbrica del libro 2 (2008), pp. 9-16.

33 Laura BARILE, "Un fenomeno di editoria popolare: le Edizione Sonzogno", in Gianfranco TORTORELLI (ed.), L'Editoria italiana tra Ottocento e Novecento. Emilia Romagna Biblioteche Archivi. Bologna, Edizioni Analisi, 1986, p. 95. 
romantica illustrata, iniciada em 1866, e, a partir de 1872, a Biblioteca romantica economica ${ }^{34}$.

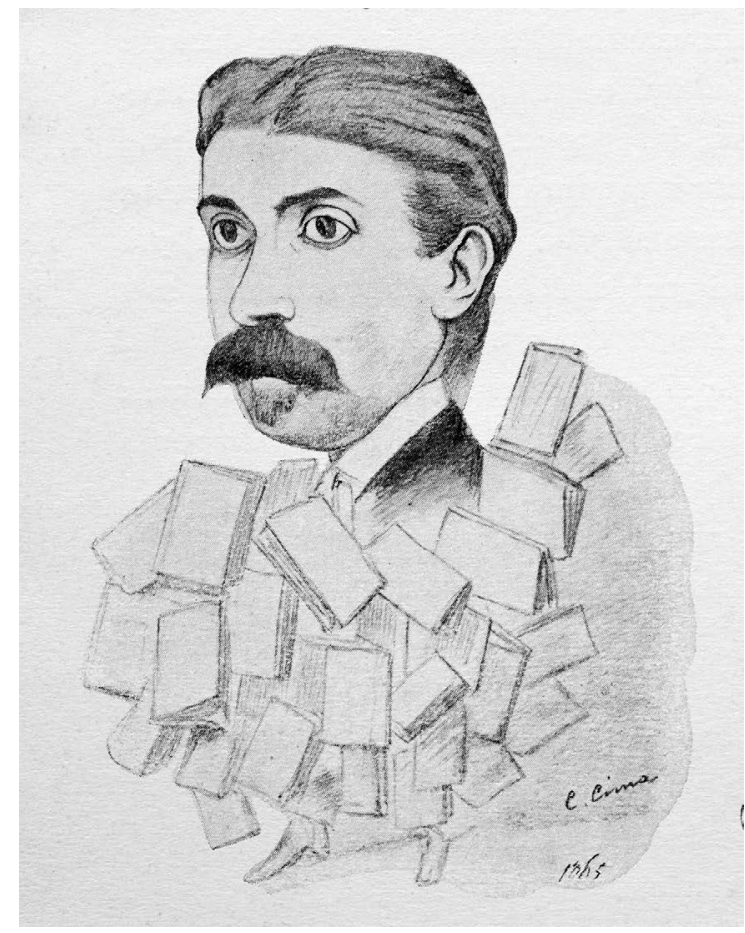

Figura 2 - Caricatura de Edoardo Sonzogno 35

$\mathrm{O}$ sucesso da editora permitiu a diversificação da sua atividade. $\mathrm{Na}$ década de 70, iniciaram-se duas outras importantes coleções: a Biblioteca Classica Economica, em 1873, e a Biblioteca del Popolo, em julho de $1875^{36}$. Note-se que a literatura atribui, incorretamente, as datas de $1873^{37}$

34 V. CAGNINELLI, op. cit.; Maria Enrica CARBOGNIN, D. LEGNANI, Anna LEVI, Lia MADORSKY, Letteratura dimenticata. Casa Editrice Sonzogno, s/d. Disponível em linha: www.letteraturadimenticata.it/Sonzogno.htm (consultado em 21/10/2010).

35 Otto CIMA, Mezzo secolo di caricatura milanese (1860-1910). Milano, Stabilimento Art Grafiche Bertarelli, 1928, p. 35.

36 ASSOCIAZIONE TIPOGRAFICO-LIBRARIA ITALIANA, Bibliografia..., p. 43.

37 Laura BARILE, “Un fenomeno...”, op. cit., p. 97. 
ou $1874^{38}$ para o início da coleção. Segundo um anúncio do editor, a 1 de julho de 1875, foram publicados os quatro primeiros números Elementi di grammatica italiana; Elementi d'aritmetica; Il mondo a volo d'ucello o geografia elementar e Compendio di cronologia - da "Nuova Biblioteca del Popolo", subintitulada "Propaganda d'instruzione". Cada número, anónimo, tinha 64 páginas, incluindo a capa e a contracapa, num formato de $11 \mathrm{~cm}$ por $16,5 \mathrm{~cm}$. Os 15 cêntimos de cada número permitiriam adquirir alternativamente três exemplares do jornal Il Secolo de quatro páginas. Os números da coleção podiam ser adquiridos avulso ou por assinatura. Um habitante no reino de Itália podia, por assinatura, adquirir a primeira série, isto é, os primeiros vinte números por três liras. Note-se que, tal como no caso da BPE, o preço da assinatura não difere da soma dos preços de capa. O custo da assinatura elevava-se a seis liras para assinantes que se encontrassem no Egito, Grécia, Portugal, Rússia, Espanha e Turquia e a sete liras para os da América, Ásia e Austrália. Os livros avulso seriam, ainda, segundo o anúncio, colocados à venda "presso tutii i Librai e rivenditori d'Italia"39. Desconhecemos, infelizmente, qualquer informação sobre a extensão e impacto desta rede de intermediários na operação da editora. Pensamos, no entanto, que o anúncio revela uma tentativa do editor de se aproximar, isto é, de "partilhar" o espaço com os possíveis leitores.

A destruição, em 1943, num bombardeamento, do arquivo da casa editorial Sonzogno limita o conhecimento das atividades desenvolvidas pela empresa ${ }^{40}$. Esperemos que o projeto, em curso, de recuperar a memória da casa editorial oitocentista através das

38 Ada Gigli MARCHETTI, "Le nuove dimensioni dell'impresa editoriale", in G. TURI, M. I. PALAZZOLO (eds.), Storia dell'editoria nell'Italia contemporanea. Saggi / [Giunti], Giunti, 1997, p. 127.

39 ASSOCIAZIONE TIPOGRAFICO-LIBRARIA ITALIANA, Bibliografia italiana: giornale dell'Associazione tipografico-libraria italiana. Anno 1872, Vol. IX da Bibliografia italiana: giornale dell'Associazione tipografico-libraria italiana. Firenze, Associazione tipografico-libraria italiana, 1877, 43.

40 L. BARILE, Le parole..., op. cit., p. 9. 
informações existentes em fontes secundárias como, por exemplo, os anúncios e as revistas bibliográficas, editadas pela empresa, possa, de futuro, trazer novos dados referentes ao real alcance geográfico da Biblioteca del Popolo ${ }^{41}$.

\section{A Bibliotheca do Povo e das Escolas e o seu modelo - semelhanças e diferenças}

Conforme anteriormente referido defendemos, neste trabalho, a tese que a Biblioteca del Popolo constituiu o modelo da BPE. No decurso da nossa investigação encontrámos várias evidências da circulação de livros da editora Sonzogno em Portugal durante o século XIX. Brito Aranha refere a tradução de Os Lusíadas de Camões, publicada pela editora em $1882^{42}$. Em nosso entender, um indício mais significativo encontra-se no espólio da biblioteca de Antero de Quental, atualmente pertencente à Biblioteca Pública de Ponta Delgada. Antero possuía a $5 .^{a}$ edição do livro Poesie de Giacomo Leopardi, publicado por Sonzogno, em 1883. O volume tem no anterrosto a dedicatória: "Ao seu querido amigo Anthero / offe. / J. d'Araújo / 16 d'Out. 83”³. Apadrinhado por Antero, Joaquim de Araújo (1858-1917) tinha publicado, em 1881, o seu

41 Silvia VALISA, "Casa editrice Sonzogno. Mediazione culturale, circuiti del sapere ed innovazione tecnologica nell'Italia unificata (1861-1900)", in A. CAESAR, J. BURNS, G. ROMANI (eds.), The Printed Media in Fin-de-siècle Italy: Publishers, Writers, and Readers. Italian perspectives. Leeds, Legenda, 2011, pp. 90-106; Silvia VALISA, "Sulla preparazione di un campione di catalogo digitale aumentato delle edizioni Sonzogno": La fabbrica del libro 2 (2013), pp. 47-54.

42 Pedro Wenceslau de Brito ARANHA, A obra monumental de Luiz de Camões: estudos bibliographicos. Lisboa, Imprensa nacional, 1886, p. 225.

43 A. RAGUSA (2016). Como exilados de um céu distante Antero de Quental e Giacomo Leopardi na 'modernidade antimoderna'. Tese de doutoramento, Faculdade de Ciências Sociais e Humanas. Universidade Nova de Lisboa, 2016, p. 62. 
primeiro livro de poesia, Lira Intima, precisamente na editora de David Corazzi, a Empreza Horas Românticas ${ }^{44}$.

\subsection{Semelhanças}

Os números das duas coleções são indistinguíveis pela sua dimensão, $11 \mathrm{~cm}$ por $16,5 \mathrm{~cm}$, número de páginas, 64, e periodicidade de publicação. Ambos têm preços muito económicos. A comparação das capas revela igualmente semelhanças gráficas e de conteúdo (figura 3).
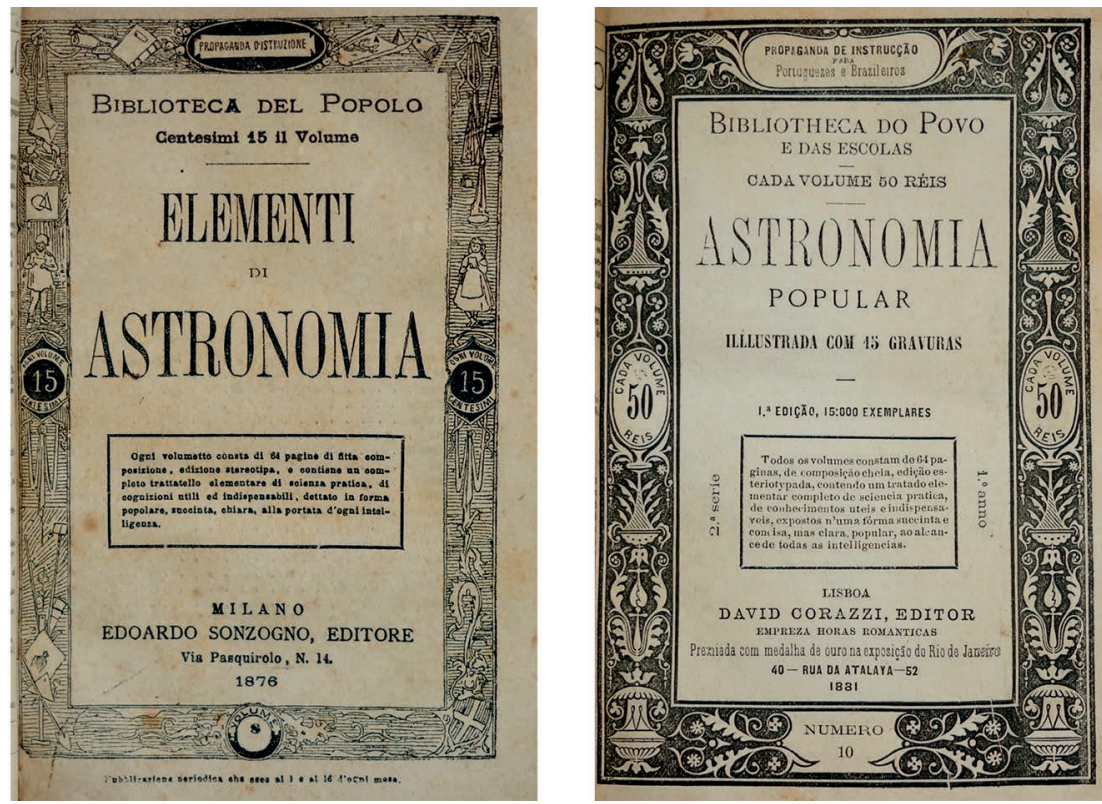

Figura 3 - Capas da edição de 1876 do n. ${ }^{\circ}$ 8, Elementi di Astronomia, da Biblioteca del Popolo ${ }^{45}$, e da primeira edição do n. ${ }^{\circ} 10$, Astronomia

Popular, da Bibliotheca do Povo e das Escolas, publicada em $1881^{46}$

${ }^{44}$ José Carlos Seabra PEREIRA, "Romantismo tardio e surto neo-romântico no fim-de-século": Humanitas 50 (1998), p. 921.

45 ANÓnImo, Elementi di Astronomia. Milano, Edoardo Sonzogno, 1876.

46 José de MELLO, Astronomia Popular. Lisboa, David Corazzi Editor - Empreza Horas Romanticas, 1881. 
O baixo custo de cada número, um elemento fundamental da estratégia comercial das editoras, aparece referido por três vezes na capa, entre os títulos da coleção e do número e em duas cartouches laterais. O texto contido na caixa retangular disposta por debaixo do título é, salvo a diferença de língua, o mesmo (figura 4), bem como os objetivos gerais das duas publicações.

Todos os volumes constam de $64 \mathrm{pa}$ ginas, de composição cheia, edição esteriotypada, contendo um tratado elementar completo de sciencia pratica, de couherimentos uteis e indispensareis, expostos n'uma fórma succinta e concisa, mas clara, popular, ao alcancede todas as intelligencias.

Ogai volumetto consta di 64 pagine di atta composizione, edizfone stereotipa, e contiene un sompleto trattatello ol omentar di soienza pratioa, di cognizioni utlli ed indispensabili, dettato in forma popolare, suceinta, ohiara, slla portata d'ogni inselligenza.

Figura 4 - Detalhe das capas apresentadas na figura 3

E se na contracapa dos Elementi di Astronomia se afirma que

Questa pubblicazione, tanto ricercata per il favoloso buon buon mercato, ha li doppio intendimento, di propagare l'instruzione generale e di far nascere l'amore allo studio nelle classi popolari così in cittá come nella champagne ${ }^{47}$.

47 ANÓNIMO, Elementi di..., op. cit. 
na da Astronomia Popular pode ler-se

Esta publicação, notavel pela sua fabulosa barateza, tem a dupla vantagem de propagar a instrucção geral e incitar ao estudo as classes populares ${ }^{48}$.

\subsection{Diferenças}

As capas revelam também diferenças de detalhe que, no nosso entender, refletem as estratégicas dos dois editores. A BPE indica na capa o número de ilustrações de cada título, a série e, até ao número 16, a tiragem. A partir do número 10 , as datas de publicação, "dias 10 e 25 de cada mez", deixam de ser referidas, o que pode revelar a dificuldade em manter este 'compromisso' com os leitores. Note-se, no entanto, que a BPE conseguiu, pelo menos numa primeira fase, entre 1881 e 1885, publicar em média dois novos títulos por mês, o que não se verificou no primeiro ano da Biblioteca del Popolo ${ }^{49}$.

Duas alterações significativas são o acrescento de "e das escolas" ao título italiano e a especificação do público a que se destinava a propaganda de instrução "Portugueses e Brazileiros" (figura 5).

${ }^{48}$ José de MELLO, Astronomia..., op. cit.

49 M. DOMINGOS, op. cit., p. 29; STABILIMENTO DEL'EDITORE EDOARDO SONZOGNO, Milano (1875). "Biblioteca del Popolo": Bibliografia italiana: giornale dell'Associazione tipografico-libraria italiana IX (24, 31 Dicembre) (1875), p. 112 e STABILIMENTO DEL'EDITORE EDOARDO SONZOGNO, "Biblioteca del Popolo": Bibliografia italiana: giornale dell'Associazione tipografico-libraria italiana, Supplemento ao n. 18 dell'Anno X (30 Settembre) (1876), p. 48. 

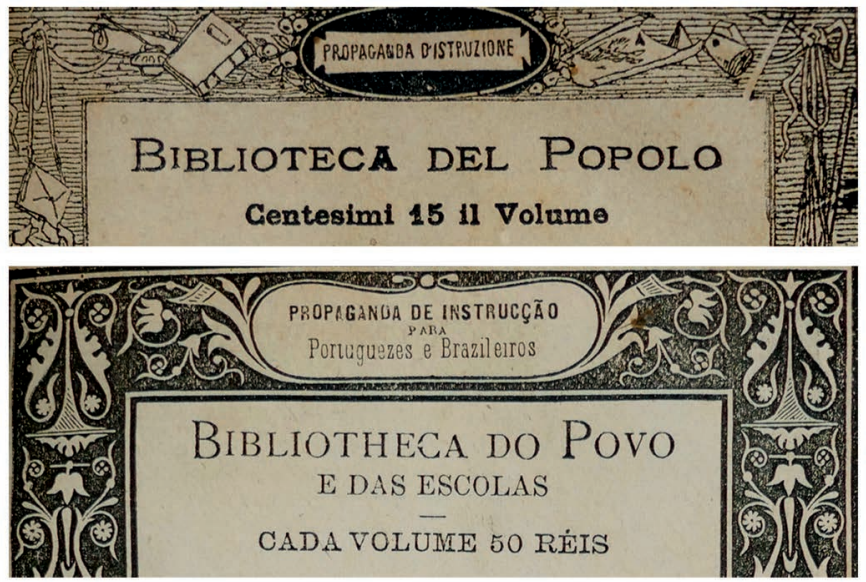

Figura 5 - Detalhe das capas apresentadas na figura 3

A alusão explícita às escolas indica que a editora pretendia que, senão todos, pelo menos alguns dos números pudessem ser utilizados no ensino. Assim, no terceiro número, Mythologia, pode ler-se que esta foi "especialmente accommodada ao ensino dos estudantes de latinidade, de poetica, de litteratura, de bellas-artes, etc"50. Se este objetivo limita, por um lado, o conteúdo, obrigando-o a respeitar o programa oficial ou parte deste, permite, por outro, o acesso a um mercado lucrativo. Em 1883, o Conselho Superior de Instrucção Pública aprovou a utilização de vários números da BPE na instrução primária e no ensino normal51.

Sabe-se que a BPE era distribuída através de uma extensa rede que se estendia do continente, às ilhas e ao Brasil ${ }^{52}$. Apesar de alguma visibilidade anterior - a Empreza Horas Romanticas obteve, por exemplo,

${ }^{50}$ Xavier da CUNHA, Mythologia. Lisboa, David Corazzi Editor - Empreza Horas Romanticas, 1881.

51 PORTUGAL. MINISTERIO DOS NEGOCIOS DO REINO, "Relação dos livros approvados pelo governo em conformidade com o parecer da junta consultiva de instrucção publica, no anno de 1882": Diário do Governo 11 (1883), p. 109.

52 M. DOMINGOS, op. cit., pp. 66-69. 
uma medalha de ouro na exposição portuguesa do Rio de Janeiro de 1879 - a estratégia para o mercado brasileiro adquiriu, em 1882, uma nova dinâmica, numa altura em que se pretendia, pelo menos legislativamente, diminuir o analfabetismo no país ${ }^{53}$. Segundo a revista $O$ Occidente,

O sr. David Corazzi um dos editores mais inteligentes, mais illustrados, e mais arrojados que Portugal tem hoje, e que alia todas as apreciaveis qualidades de um perfeito cavalheiro delicadissimo e brioso, com as d'um negociante laborioso e habil, vae tentar uma empresa corajosa que estamos certos lhe dará os melhores resultados e explorar em grande escala o mercado literário do Brazil, fundando no Rio de Janeiro uma filial da sua acreditada e já importantíssima casa editora. O sr. José Maria de Mello, que vae encarregado de montar no Rio essa filial e de a dirigir, é um rapaz muito inteligente, muito activo a quem a Empreza Horas Romanticas deve, desde os seus princípios, uma cooperação leal e solicita e que empregará agora todos os seus esforços para a fazer desenvolver e progredir rapidamente no Brazil ${ }^{54}$.

José Maria de Mello (?-?) era amigo de David Corazzi e autor do décimo número da BPE, Astronomia Popular. Mello seria, mais tarde, gerente da empresa sucessora da Empreza Horas Romanticas, a Companhia Nacional Editora. A partir do número 37, de 1882, passa a constar nas capas dos números da BPE a informação "Filial no Brasil: 40, R. da Quitanda, Rio de Janeiro" 55 . Estudos recentes indicam uma boa difusão da BPE no Brasil ${ }^{56}$.

53 X. da CUnHA, Mythologia, op. cit.; M. Domingos, op. cit.; Carlos COSTA, A Revista no Brasil do Século XIX. São Paulo, Alameda Casa Editorial, 2012, pp. 72-83.

54 G. LOBATO, "Chronica Occidental”: O Occidente 125 (1882), pp. 129-130.

55 F. A. C. SOARES, Tactica e Armas de Guerra. Lisboa, David Corazzi Editor Empreza Horas Romanticas, 1882.

56 J. NASCIMENTO, V. SANTOS, op. cit.; G. M. VENÂNCIO, "Lisboa-Rio...", op. cit. 
Por último, encontramos uma diferença importante na metodologia utilizada pela editora portuguesa na gestão das diferentes séries. A dimensão de uma série foi reduzida de 20 para 8 números e estimulou-se a sua encadernação. Os números da BPE incluem, ao contrário da coleção italiana, a informação da série na capa e a partir da $6 .^{a}$ série $^{57}$, publicada em 1882, a editora ofereceu "cada serie de 8 volumes, cartonada em percalina, 500 réis; capas separadas para cartonar cada serie, 100 réis" 58 . Desta forma, cada número pode ser interpretado como um fascículo de um livro (a série). A biblioteca económica do "operario, o estudante, o chefe de familia ou o professor" seria constituída pelo conjunto dos livros/séries ${ }^{59}$. A publicação de prólogos e/ou erratas de uma série remete, de novo, para a sua encadernação. Assim, enquanto existem várias coleções quase completas da BPE, quer em Portugal, quer no Brasil, quer em bibliotecas públicas, quer em coleções privadas, nas pesquisas efetuadas nos catálogos de várias bibliotecas italianas não encontrámos nenhuma série encadernada da Biblioteca del Popolo nem indicação de que esta pudesse existir. A encadernação das 1280 páginas de uma série da Biblioteca del Popolo parece-nos impraticável. Os volumes encadernados que encontrámos à venda contêm, habitualmente, 8 a 10 números selecionados, por vezes, pela coerência temática dos seus conteúdos.

\section{Conclusões}

Enquadrando-se nas dinâmicas editoriais do século XIX, a Biblioteca del Popolo é dada à estampa a partir de 1875, em Milão.

57 É possível que esta iniciativa seja de data anterior, visto que existe uma errata da $2 .^{a}$ série, datada de 1881.

58 ANÓNIMO, Biblioteca do Povo e das Escolas. Sexta Serie. Lisboa, David Corazzi Editor. Empreza Horas Romanticas, 1883.

59 ANÓNIMO, Biblioteca do Povo..., op. cit. 
Seis anos mais tarde, uma publicação com as mesmas características, a BPE, inspira-se neste modelo estrangeiro. Como vimos, a publicação portuguesa partilhava o ideal de providenciar instrução às classes populares a preços económicos, e tinha o mesmo número de páginas e dimensão igual à sua congénere italiana. $\mathrm{O}$ grafismo e o conteúdo das capas das duas publicações é, também, muito semelhante. Ambas as 'bibliotecas' foram sucessos comerciais durante vários anos, apesar de nenhuma ter conseguido manter a ambiciosa taxa de publicação de dois números por mês ${ }^{60}$.

Uma análise preliminar das publicações indica que os títulos da BPE não são traduções dos da Biblioteca del Popolo. Nos primeiros anos, as temáticas de ciências naturais e exatas parecem ter uma maior expressão na BPE, possivelmente como consequência da formação médica e opções de Xavier da Cunha, o diretor da coleção. Espera-se que a conclusão do estudo comparativo em curso permita esclarecer estas duas questões.

No decurso desta investigação encontrámos, ainda, outra ligação entre as editoras da BPE e da Biblioteca del Popolo. Em 1882, Sonzogno inicia a publicação de uma nova coleção de literatura, a Biblioteca Universale. Antica e moderna, em que cada pequeno volume tinha um preço de "25 centesimi" 61 . Os Lusíadas de Camões, anteriormente referidos, correspondiam aos números 11 e 12 dessa coleção. Seis anos mais tarde, David Corazzi inicia a publicação da sua Bibliotheca Universal. Antiga e Moderna ${ }^{62}$. Verifica-se, de novo, uma inspiração da publicação italiana no nome, conteúdo e capa de brochura da coleção portuguesa (figura 6). A primeira edição do volume Escaravelho de ouro foi publicada, em 1889, pela

60 STABILIMENTO DEL'EDITORE EDOARDO SONZOGNO, "Pubblicazione educative": Il Secolo 21-22 Settembro (1885), p. 4.

61 Silvia VALISA, "Casa editrice...", op. cit., p. 98.

62 ANÓNIMO, "Publicações": O Occidente 11 (329, 11 de Fevereiro) (1888), p. 40. 
Companhia Nacional Editora, sucessora, a partir de 1888, da empresa de Corazzi. A capa da brochura indica uma 2. ${ }^{a}$ edição de "A Editora". Um catálogo de 1903 intitulado "A Editora - Antiga Casa David Corazzi" estabelece a relação entre ambas as empresas ${ }^{63}$.
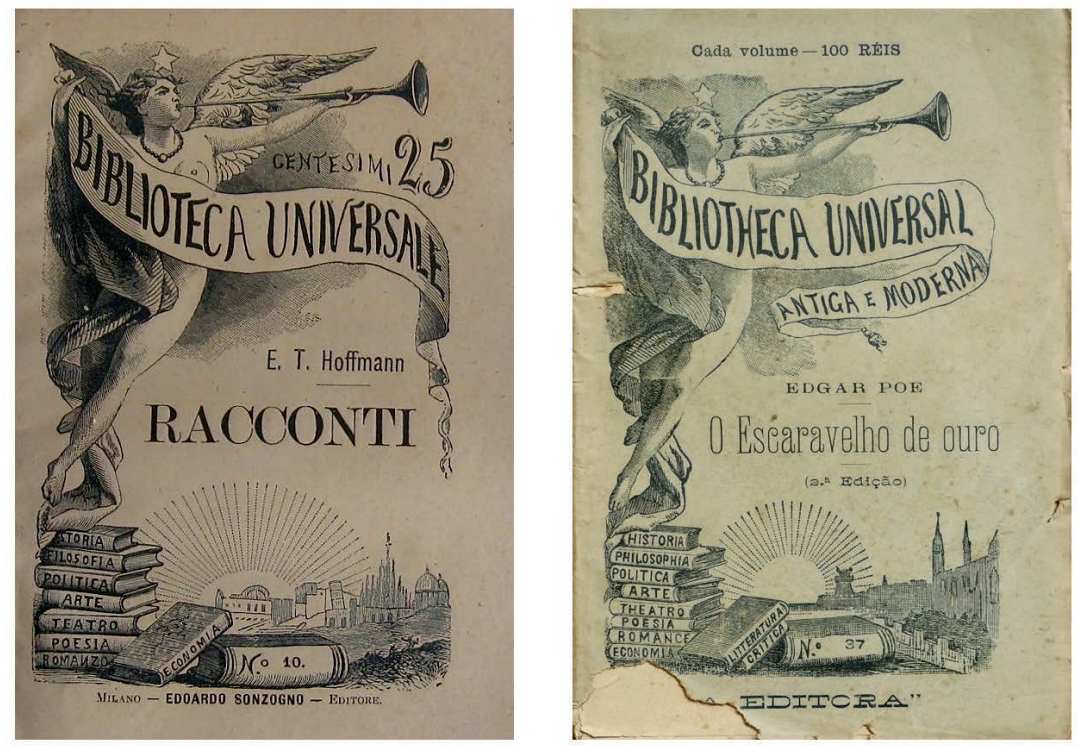

Figura 6 - Capas da edição de 1882 do n. ${ }^{\circ} 10$ da Biblioteca Universale e do n. ${ }^{\circ} 37$ da Bibliotheca Universal ${ }^{64}$

Em resumo, face às semelhanças encontradas entre a Biblioteca del Popolo e a Bibliotheca do Povo e das Escolas, concluímos ser improvável o aparecimento e desenvolvimento independente desta última. As principais diferenças detetadas resultam, no nosso entender, de opções estratégicas do editor português que pretendia tirar partido da expansão dos sistemas de ensino e aceder ao mercado brasileiro.

63 M. DOMINGOS, Estudos.., op. cit., p. 30

${ }^{64}$ E. T. HOFFMANN, Racconti. Milano, Edoardo Sonzogno, Editore, 1882; Edgar Allen POE, O Escaravelho de ouro. Lisboa, A Editora, s/d. 


\section{Referências bibliográficas}

ANÓNIMO, Elementi di Astronomia. Milano, Edoardo Sonzogno, 1876.

ANÓNIMO, "Astronomia popular": O Primeiro de Janeiro 13 de Julho (1881), p. 2.

ANÓNIMO, Biblioteca do Povo e das Escolas. Sexta Serie. Lisboa, David Corazzi Editor. Empreza Horas Romanticas, 1883.

ANÓNIMO, "Publicações": O Occidente 11 (329, 11 de Fevereiro) (1888), p. 40.

ANÓNIMO, "Comemorações do XXV anno do Occidente": O occidente: revista illustrada de Portugal e do estrangeiro XXV (829-830, 10 e 20 de Janeiro) (1902), pp. 2-14.

ARANHA, Pedro Wenceslau de Brito, A obra monumental de Luiz de Camões: estudos bibliographicos. Lisboa, Imprensa nacional, 1886.

ASSOCIAZIONE TIPOGRAFICO-LIBRARIA ITALIANA, Bibliografia italiana: giornale dell'Associazione tipografico-libraria italiana. Anno 1872, Vol. IX da Bibliografia italiana: giornale dell'Associazione tipografico-libraria italiana. Firenze, Associazione tipografico-libraria italiana, 1877.

BARBIER, Frédéric, "Libraires et colporteurs", in Roger CHARTIER, Henri-Jean MARTIN (eds.), Histoire de l'édition française, Vol. 3. Paris, Fayard, Cercle de la Librarie, 1990, pp. 256-302.

BARILE, Laura, "Un fenomeno di editoria popolare: le Edizione Sonzogno", in Gianfranco TORTORELLI (ed.), L'Editoria italiana tra Ottocento e Novecento. Emilia Romagna Biblioteche Archivi. Bologna, Edizioni Analisi, 1986, pp. 95-105.

BARILE, Laura, Le parole illustrate: Edoardo Sonzogno editore del popolo. Ghirlandina. Incontri con l'Ottocento. Modena, Mucchi Editore, 1994.

BEAVAN, Iain, "Advertising Judiciously: Scottish Nineteenth-Century Publishers and the British Market" in Peter ISAAC, Barry MCKAY (eds.), The Mighty Engine: The printing Press \& its Impact. Winchester, St Paul's Bibliographies and Delaware, Oak Knoll Press, 2000, pp. 69-78.

BÉGUET, Bruno, "La vulgarisation scientifique au XIX ${ }^{\mathrm{e}}$ siècle", in Bruno BÉGUET, Maryline CANTOR, Ségolène LE MEN (eds.), La Science pour Tous. Paris, Réunion des Musées Nationaux, 1994, pp. 5-48.

BONIFÁCIO, Vitor; MALAQUIAS, Isabel; FERNANDES João, “Ernesto Vasconcellos' Astronomia Photographica: the earliest popular book on astronomical photography?": Journal of Astronomical History and Heritage 11 (2008), pp. 116-123.

BONIFÁCIO, Vitor, Da Astronomia à Astrofísica: A perspectiva portuguesa. Tese de Doutoramento, Universidade de Aveiro, Departamento de Física, 2009.

BONIFÁCIO, Vitor; MALONEK, Helmuth, "Os inesperados livros de Matemática da "Bibliotheca do Povo e das Escolas"”: Suplemento do Boletim da Sociedade Portuguesa de Matemática 69 (2013), pp. 35-37.

CAGNINELLI, Viola, "Edoardo Sonzogno rappresentante italiano della Société des gens de lettres (1872-1878)": La fabbrica del libro 2 (2008), pp. 9-16.

CARBOGNIN, Maria Enrica; LEGNANI, D.; LEVI, Anna; MADORSKY, Lia, Letteratura dimenticata. Casa Editrice Sonzogno, s/d. Disponível em linha: www. letteraturadimenticata.it/Sonzogno.htm (consultado em 21/10/2010). 
CIMA, Otto, Mezzo secolo di caricatura milanese (1860-1910). Milano, Stabilimento Arti Grafiche Bertarelli, 1928.

COSTA, Carlos, A Revista no Brasil do Século XIX. São Paulo, Alameda Casa Editorial, 2012.

CUNHA, Xavier da, Historia de Portugal, 2. ${ }^{a}$ edição. Lisboa, David Corazzi Editor - Empreza Horas Romanticas, 1881.

CUNHA, Xavier da, Mythologia. Lisboa, David Corazzi Editor - Empreza Horas Romanticas, 1881.

CUNHA, Xavier da, "Duas palavras ao leitor", in Biblioteca do Povo e das Escolas. Terceira Serie. Lisboa, David Corazzi - Editor. Empreza Horas Romanticas, 1882.

CUNHA, Xavier da, "Quatro paginas de prólogo", in Biblioteca do Povo e das Escolas. Sexta Serie. Lisboa, David Corazzi - Editor. Empreza Horas Romanticas, 1883.

DOMINGOS, Manuela, Estudos de Sociologia da Cultura. Livros e Leitores do Século XIX. Lisboa, Instituto Português do Ensino à Distância, 1985.

HOMBURG, Ernst, "From Chemistry for the People to the Wonders of Technology: The Popularization of Chemistry in the Netherlands during the Nineteenth Century": Hyle - International Journal for Philosophy of Chemistry 12(2) (2006), pp. 163-191.

HOFFMANN, E. T., Racconti. Milano, Edoardo Sonzogno Editore, 1882.

HOMEM, António Pedro Barbas, Manuel Joaquim Pinheiro Chagas, 1842-1895: uma biografia. Lisboa, Colecção Parlamento, Assembleia da República, 2012.

LACERDA, João Cesario de, Hygiene, Lisboa, David Corazzi Editor - Empreza Horas Romanticas, 1881.

LIGHTMAN, Bernard, "The Voices of Nature: Popularizing Victorian Science", in Bernard LIGHTMAN (ed.), Victorian Science in Context. Chicago, The University of Chicago Press, 1997, pp. 187-211.

LIMA, Magalhães, "David Corazzi": Commercio e Industria - Sciencias, Artes e Letras 6 (1880), p. inum.

LOBATO, G., "Chronica Occidental”: O Occidente 125 (1882), pp. 129-130.

LYONS, Martyn, "Les best-sellers", in Roger CHARTIER, Henri-Jean MARTIN (eds.), Histoire de l'édition française, Vol. 3. Fayard, Cercle de la Librarie, 1990, pp. 409-448,

LYONS, Martyn, "New Readers in the Nineteenth Century: Women, Children, Workers", in Guglielmo CAVALLO, Roger CHARTIER (eds.), A History of Reading in the West. Cambridge, Blackwell, 2003, pp. 313-344.

MARCHETTI, Ada Gigli, "Le nuove dimensioni dell'impresa editoriale", in G. TURI, M. I. PALAZZOLO (eds.), Storia dell'editoria nell'Italia contemporanea. Saggi / [Giunti], Giunti, 1997, pp. 115-164.

MARX, Karl, Le capital. Paris, M. Lachâtre, 1872.

MATOS, Ana Cardoso de, "Os agentes e meios de divulgação científica e tecnológica em Portugal no século XIX”: Scripta Nova 69 (2000). Disponível em linha: http:// www.ub.edu/geocrit/sn-69-29.htm (consultado em 15/06/2018).

MELlO, José de, Astronomia Popular. Lisboa, David Corazzi Editor - Empreza Horas Romanticas, 1881. 
MOLLIER, Jean-Yves, "La construction du système éditorial français et son expansion dans le monde du XVIII ${ }^{\mathrm{e}}$ au XX $\mathrm{XX}^{\mathrm{e}}$ siècle", in Jacques MICHON, Jean-Yves MOLLIER (eds.), Les mutations du livre et de l'édition dans le monde du XVIII siècle à l'an 2000. Paris, L'Harmattan, 2003, pp. 47-72.

NABO, Olímpia de J. de B. M., Educação e difusão da ciência em portugal A 'Bibliotheca do Povo e das Escolas' no Contexto das Edições Populares do Século XIX. Tese de Mestrado, Escola Superior de Educação do Instituto Politécnico de Portalegre, 2012.

NASCIMENTO, Jorge, "Nota prévia sobre a palavra impressa no Brasil no século XIX: a biblioteca do povo e das escolas": Horizontes 19 (2001), pp. 11-27.

NASCIMENTO, Jorge; SANTOS, V., "Geografia geral para portugueses e brasileiros: a biblioteca do povo e das escolas": Revista da Faced 10 (2006), pp. 141-158.

NUNES, Maria de Fátima, "A Bibliotheca do Povo e das Escolas: Fonte para a História da Ciência”, in Mari Álvarez LIRES, Ánxela Bugallo RODRÍGUEZ, José $\mathbf{M}^{a}$ FERNANDÉZ, Rafael Sisto EDREIRA, X. Carlos Valle PÉREZ (coord.), Estudios de Historia das Ciencias e das Técnicas, vol. 1. Vigo, Deputacíon Provincial. Servicio de Publicacións, 2001, pp. 221-231.

PEREIRA, José Carlos Seabra, "Romantismo tardio e surto neo-romântico no fim-de-século": Humanitas 50 (1998), pp. 915-962.

POE, Edgar Allen, O Escaravelho de ouro. Lisboa, A Editora, s/d.

PORTUGAL. MINISTERIO DOS NEGOCIOS DO REINO, "Relação dos livros approvados pelo governo em conformidade com o parecer da junta consultiva de instrucção publica, no anno de 1882": Diário do Governo 11 (1883), p. 109.

RAGUSA, A., Como exilados de um céu distante Antero de Quental e Giacomo Leopardi na 'modernidade antimoderna'. Tese de doutoramento, Faculdade de Ciências Sociais e Humanas. Universidade Nova de Lisboa, 2016.

SANTOS, M., "A elite intelectual e a difusão do livro nos meados do século XIX": Análise Social XXVII (116-117) (1992), pp. 539-546.

SHEETS-PYENSON, S., "Popular Science Periodicals in Paris and London: the Emergence of a Low Scientific Culture, 1820-1875": Annals of Science 42(6) (1985), pp. 549-572.

SOARES, F. A. C., Tactica e Armas de Guerra. Lisboa, David Corazzi Editor - Empreza Horas Romanticas, 1882.

STABILIMENTO DEL'EDITORE EDOARDO SONZOGNO, "Pubblicazione educative": Il Secolo 21-22 Settembro (1885), p. 4.

STABILIMENTO DEL'EDITORE EDOARDO SONZOGNO, "Biblioteca del Popolo": Bibliografia italiana: giornale dell'Associazione tipografico-libraria italiana IX (24, 31 Dicembre) (1875), p. 112.

STABILIMENTO DEL'EDITORE EDOARDO SONZOGNO, "Biblioteca del Popolo": Bibliografia italiana: giornale dell'Associazione tipografico-libraria italiana, Supplemento ao n. 18 dell'Anno X (30 Settembre) (1876), p. 48.

THIESSE, Anne-Marie, Le roman du quotidian. Paris, Éditions du Seuil, 2000.

TORGAL, Luís Reis, "A instrução pública”, in José MATTOSO (ed.), História de Portugal, Vol. 5. Círculo de Leitores, 1993, pp. 609-651.

VALISA, Silvia, "Casa editrice Sonzogno. Mediazione culturale, circuiti del sapere ed innovazione tecnologica nell'Italia unificata (1861-1900)", in A. CAESAR, J. BURNS, 
G. ROMANI (eds.), The Printed Media in Fin-de-siècle Italy: Publishers, Writers, and Readers. Italian perspectives. Leeds, Legenda, 2011, pp. 90-106.

VALISA, Silvia, "Sulla preparazione di un campione di catalogo digitale aumentato delle edizioni Sonzogno": La fabbrica del libro 2 (2013), pp. 47-54.

VENÂNCIO, Giselle M., "Lisboa-Rio de Janeiro-Fortaleza: os caminhos da coleção Biblioteca do Povo e das Escolas, traçados por David Corazzi, Francisco Alves e Gualter Rodrigues": Cultura 21 (2005), pp. 185-204. 